\title{
THE MODULAR INTERPOLATION INEQUALITY IN SOBOLEV SPACES WITH VARIABLE EXPONENT ATTAINING THE VALUE 1
}

\author{
FLAVIA GiAnNETTI
}

Abstract. We prove a modular type interpolation inequality for functions belonging to Sobolev spaces with variable exponent attaining the value 1 . The approach combines the original proof of the interpolation inequality by Nirenberg [19] with an inequality for averages over balls, avoiding the use of the norm interpolation inequality for variable exponent Sobolev spaces, known for exponents whose infimum is greater than 1 .

Mathematics subject classification (2010): 26D10, 46E30, 46E35.

Keywords and phrases: Variable Sobolev space, variable Lebesgue space, interpolation inequalities, modular inequalities.

\section{REFERENCES}

[1] R. Adams, Sobolev spaces, Academic Press, New York-London, 1975.

[2] C. CAPONE, D.CRUZ-URIBE AND A.FiorenZA, Fractional maximal operators and fractional integrals on $L^{p(\cdot)}$, Rev. Mat. Iberoam., 23, 3 (2007), 743-770.

[3] D.Cruz-Uribe And A.Fiorenza, Approximate identities in variable $L^{p}$ spaces, Math. Nachr., 280, 3 (2007), 256-270.

[4] D.Cruz-Uribe, A.Fiorenza And C.J.Neugebauer, The maximal function on variable $L^{p}$ spaces, Ann. Acad. Sci. Fenn. Math., 28 (2003), 223-238, and 29 (2004), 247-249.

[5] P.Cianci AND F.F.Nicolosi, A weighted interpolation inequality with variable exponent of the Nirenberg Gagliardo kind, Nonlinear Anal., 71 (2009), 5915-5929.

[6] L. Diening, Maximal functions on generalized $L^{p(x)}$ spaces, Math. Inequal. Appl., 7, 2 (2004), $245-253$.

[7] G. EHRLING, On a type of eigenvalue problem for certain elliptic differential operators, Math. Scand., 2 (1954), 267-285.

[8] X.-L. Fan And D.Zhao, On the spaces $L^{p(x)}(\Omega)$ and $W^{m, p(x)}(\Omega)$, J. Math. Anal. Appl., 263, 2 (2001), 424-446.

[9] E. Gagliardo, Ulteriori proprietá di alcune classi di funzioni in piú variabili, Ricerche Mat., 8 (1959), 24-51.

[10] P. HaRJUlehto AND P. HÄstÖ, An overview of variable exponent Lebesgue and Sobolev spaces, Future trends in geometric function theory, Rep. Univ. Jyväskilä Dep. Math. Stat. 92, Univ. Jyväskilä, Jyväskilä (2003), 85-93.

[11] T.Kopaliani AND G.Chelidze, Gagliardo-Nirenberg type inequality for variable exponent Lebesgue spaces, J. Math. Anal. Appl., 356, 1 (2009), 232-236.

[12] O. KovÁČIIK AND J. RÁKosníK, On spaces $L^{p(x)}$ and $W^{k, p(x)}$, Czechoslovak Math. J., 41(116), 4 (1991), 592-618.

[13] A.Lerner, On modular inequalities in variable $L^{p}$ spaces, Arch. Math.(Basel), 85, 6 (2005), 538543.

[14] J.L. Lions, Lectures on elliptic partial differential equations, Tata Institute, Bombay, 1957.

[15] L. Maligranda, Orlicz spaces and interpolation, Seminários de Matemática, Univ. Estadual de Campinas, 1989.

[16] V.G. MAZ'JA, Sobolev spaces, Springer Verlag, Berlin, 1985.

[17] J.MusielaK, Orlicz spaces and modular spaces, Springer Verlag, Berlin, 1983. 
[18] J.NECǍs, Les méthodes directes en théorie des équations elliptiques, Masson et C. Éditeurs, Paris, 1967.

[19] L. NiRenberg, Remarks on strongly elliptic PDE, Comm. Pure Appl. Math., 8 (1955), $649-675$.

[20] S.G. SAMKO, On a progress in the theory of Lebesgue spaces with variable exponent: maximal and singular operators, Integral Tranforms Spec. Funct., 16, 5-6 (2005), 461-482.

[21] A. ZANG AND Y.FU, Interpolation inequalities for derivatives in variable exponent Lebesgue-Sobolev spaces, Nonlinear Anal., 69, 10 (2008), 3629-3636. 